\title{
Towards Serverless NFV for 5G Media Applications
}

\author{
David Breitgand \\ IBM Research - Haifa, Israel \\ davidbr@il.ibm.com \\ David Griffin \\ University College London, UK \\ d.griffin@ucl.ac.uk \\ Nikolaos Zioulis \\ Centre for Research and \\ Technology Hellas,Greece \\ nzioulis@iti.gr
}

\author{
Avi Weit \\ IBM Research - Haifa, Isreal \\ weit@il.ibm.com \\ Ugur Acar \\ Netas, Turkey \\ uacar@netas.com.tr
Pasquale Andriani
Engineering Ingegneria
Informatica, Italy \\ pasquale.andriani@eng.it
}

\author{
Stamatia Rizou \\ Singular Logic, Greece \\ srizou@singularlogic.eu \\ Gino Carrozzo \\ Nextworks, Italy \\ g.carrozzo@nextworks.it \\ Francesco Iadanza \\ Engineering Ingegneria \\ Informatica, Italy \\ francesco.iadanza@eng.it
}

\begin{abstract}
The advent of virtualization and IaaS have revolutionized the telecom industry via SDN/NFV. A new wave of cloudnative PaaS promises to further improve SDN/NFV performance, portability, and cost-efficiency. In this poster, we highlight a work in progress being done in the 5G-MEDIA project [2], which pioneers the application of the serverless paradigm to NFV in the context of media intensive applications in 5G networks. Motivational use cases include tele-immersive gaming, mobile journalism and UHD content distribution. For example, consider a next-gen e-sport, in which bouts between gamers last only a few minutes. FaaS offers a clear cost-efficiency benefit for hosting such applications. An architecture is shown in Fig. 1. It includes i) an Application/Service Development Kit (SDK) to enable access to media applications development tools; ii) a Service Virtualization Platform (SVP) to run the ETSI MANO framework, the Media Service MAPE optimization component and the VIM and WIM plugins to enable NFVIs integration; iii) different NFVIs to execute media-specific VNFs. FaaS VIM is implemented for integration of FaaS with the rest of the MANO stack. It allows mixing FaaS and "regular" VNFs within the same media forwarding graph. For reference implementation,
\end{abstract}

Permission to make digital or hard copies of all or part of this work for personal or classroom use is granted without fee provided that copies are not made or distributed for profit or commercial advantage and that copies bear this notice and the full citation on the first page. Copyrights for components of this work owned by others than the author(s) must be honored. Abstracting with credit is permitted. To copy otherwise, or republish, to post on servers or to redistribute to lists, requires prior specific permission and/or a fee. Request permissions from permissions@acm.org. SYSTOR, 2018, Haifa, Israel

(C) 2018 Copyright held by the owner/author(s). Publication rights licensed to Association for Computing Machinery. ACM ISBN 123-4567-24-567/08/06 ..\$15.00 https://doi.org/10.475/123_4

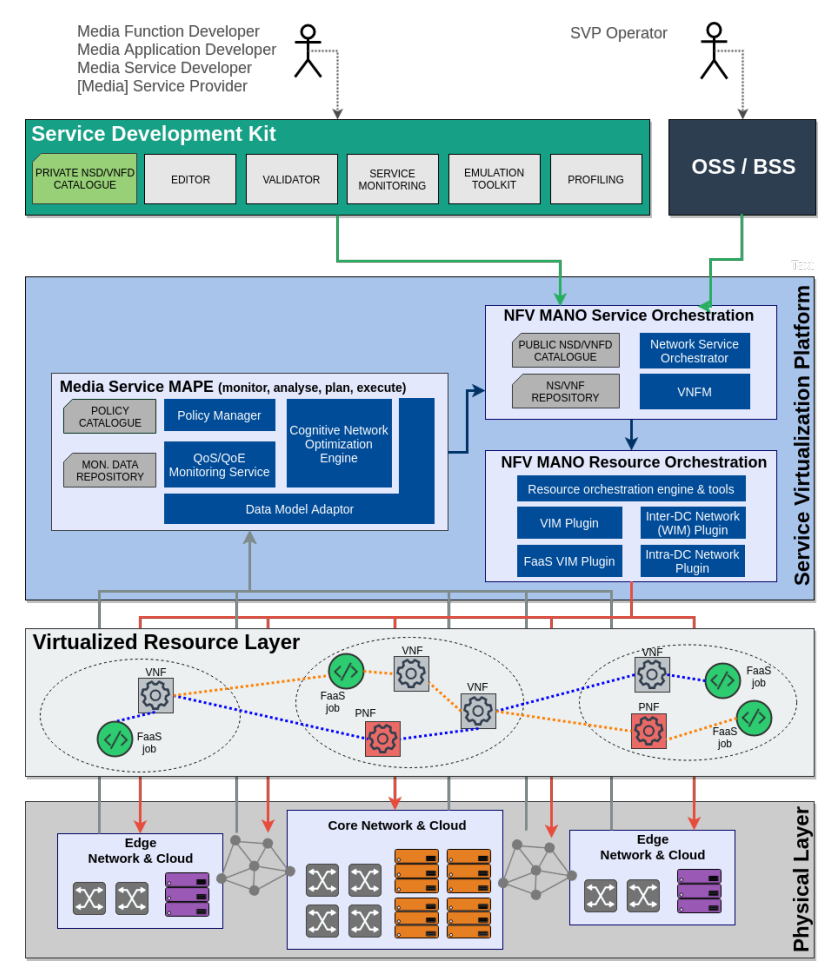

Figure 1: 5G-MEDIA Architecture

Apache OpenWhisk [1] and Kubernetes are used. The main challenge is extending the programming model to support groups of actions communicating over a network, while retaining the simplicity of FaaS. The project is supported by EU H2020 R\&I program (Grant Agreement No 761699).

\section{REFERENCES}

[1] 2018. Apache OpenWhisk. https://openwhisk.apache.org/. (2018).

[2] Stamatia Rizou et.al. 2018. A service platform architecture enabling programmable edge-to-cloud virtualization for the 5G Media industry. 